\title{
Modified Flapless and Sutureless Technique of Scleral Fixation of Intraocular Lens in the Management of Dislocation of Crystalline Lens Following Closed Globe Injury
}

Naresh Babu Kannan

Aravind Eye Hospital

Sagnik Sen ( $\nabla$ riksag@gmail.com )

Aravind Eye Hospital https://orcid.org/0000-0001-5835-5371

Gauri Khare

Aravind Eye Hospital

Obuli Ramachandran

Aravind Eye Hospital

Kim Ramasamy

Aravind Eye Hospital

\section{Research Article}

Keywords: SFIOL, pars plana vitrectomy, PPV, aphakia, closed globe injury

Posted Date: March 15th, 2021

DOI: https://doi.org/10.21203/rs.3.rs-230197/v1

License: (c) (i) This work is licensed under a Creative Commons Attribution 4.0 International License.

Read Full License 


\section{Abstract \\ Purpose}

To evaluate the outcomes of management of subluxation of lens following closed globe injury with a modified technique of scleral fixation of intraocular lens (SFIOL).

\section{Methods}

This prospective longitudinal study evaluated eyes with subluxation of crystalline lens which underwent combined pars plana vitrectomy (PPV), lensectomy (PPL) along with a sutureless and flapless technique of SFIOL implantation. We documented clinico-demographic data of all eyes at baseline and final anatomical and visual outcomes at 6 months after the surgery.

\section{Results}

We evaluated 113 eyes of 113 subjects with a mean age of $51.5+-15.7$ years. $48.7 \%$ of eyes had a partial subluxation of lens from patellar fossa, while $51.3 \%$ of eyes had complete subluxation. After 6 months, the baseline visual acuity of $1.40+-0.66 \log$ MAR (Snellen equivalent $=6 / 180$ ) increased to 0.23 +0.27 (Snellen equivalent $=6 / 9)(p<0.001$ ). Eyes having $V A<6 / 36$ reduced from $79.6 \%$ at baseline to $4.4 \%$ at final follow-up $(p<0.001)$. Eyes having VA> $=6 / 12$ increased from $8.8-85.8 \%(p<0.001)$. The proportion of eyes having raised intraocular pressure reduced from $20.4-1.8 \%$ at final follow-up $(p<$ 0.001). The most common cause of subnormal visual gain was macular degeneration secondary to commotio retinae.

\section{Conclusion}

Subluxation of lens is a common entity faced by ophthalmologists across the world. Lensectomy with PPV and SFIOL is an effective method of refractive rehabilitation for subluxation eyes following closed globe injury. Visual prognosis may still depend upon the initial insult on the retina.

\section{Introduction}

Subluxation of lens is a common entity faced by ophthalmologists anywhere in the world, and these clinical scenarios may be challenging for any surgeon depending on the varied presentations and skillsets each surgeon has acquired. Quite often patients may have posterior lens dislocation into the vitreous cavity and anterior segment surgeons are not trained or do not have the surgical setup to handle such cases, hence these cases are managed by posterior segment vitreo-retinal surgeons. Mild cases of subluxation may be managed with implantation of intraocular lens (IOL) in the bag along with capsular supportive devices [1]. However, with higher degrees of subluxation from the patellar fossa, the capsular 
bag cannot be salvaged and needs to be removed. In these cases of aphakia, conventional posterior chamber IOL (PCIOL) implantation in the bag or sulcus is not an option, hence iris fixated IOLs, anterior chamber IOLs and scleral-fixated IOLs have become popular. In this study, we describe management outcomes of SFIOL implantation after dislocation of lens in the setting of closed globe injury, with our modified technique which we have employed previously for ectopia lentis and spherophakia eyes $[2,3]$.

\section{Methods}

This prospective study was conducted at a tertiary care centre in South India after obtaining proper ethical clearance from the Institutional Review Board and the study adhered to the tenets of the Declaration of Helsinki. All subjects had given written informed consent before inclusion in the study. The study included all patients with the history of ocular trauma associated with subluxation or dislocation of the crystalline lens from the patellar fossa anteriorly into the anterior chamber or posteriorly towards the vitreous cavity, in which placement of a PCIOL in the bag was deemed unfit based on physician's discretion. All patients underwent pars plana vitrectomy with lensectomy and sutureless scleral fixation intraocular lens implantation. Data recorded for all patients included age, gender, mode of trauma, baseline visual acuity, intraocular pressure (IOP), ocular clinical presentation, refractive error, final postoperative visual outcome, IOL status and complications noted postoperatively. All patients were followed up for a duration of 6 months to evaluate the change in outcomes.

\section{Protocol For Surgery}

All eyes underwent 23-gauge (G) pars plana vitrectomies along with lensectomy and SFIOL implantation. All surgeries were performed under strict asepsis using a uniform surgical technique. Following peritomy, two partial thickness scleral pockets were made parallel to limbus, 180 degrees apart, 1.5-2 $\mathrm{mm}$ away from limbus with 23G microvitreoretinal (MVR) blade. Two sclerotomies were made close to the scleral pockets with a $24 \mathrm{G}$ needle for externalization of IOL haptics.

Lensectomy and core vitrectomy were performed, followed by entry into the anterior chamber (AC) through a superior scleral tunnel with the help of a keratome blade. A three-piece non-foldable IOL (Aurolab, Madurai, India) was placed into the AC through the tunnel. The leading haptic tip was pulled out through sclerotomy with a $23 \mathrm{G}$ end grasping forceps (Alcon Laboratories, Fort Worth, Texas, USA) and tucked into the scleral pocket. The second haptic was similarly grasped and pulled out through the second sclerostomy and tucked into the scleral pocket. After ensuring lens centration, vitrectomy ports, scleral tunnel and conjunctiva were sutured, with the globe saline filled. In eyes displaying postoperative IOL decentration, a localized peritomy was performed around the scleral pockets and the length of haptic adjusted. At the end of all surgeries, ofloxacin $0.3 \%$ eye ointment was put in the conjunctival cul-de-sac. All patients were routinely prescribed a topical combination therapy of antibiotics (ofloxacin $0.3 \%$ ) and steroid (prednisolone acetate suspension 1\%) for a period of 6 weeks. Postoperatively, slit lamp examination was performed at each follow-up to look for corneal clarity, anterior chamber integrity and 
status of the IOL. Intraocular pressure was measured using non-contact tonometer and all patients underwent retinoscopy. Postoperative complications if detected in any eye was treated accordingly.

\section{Statistical analysis}

Data were analyzed using SPSS for Windows software (version 20.0, International Business Machines Corp.). Visual acuity was converted from Snellen letters to logarithm of minimum angle of resolution (logMAR) for analysis. Data normality was checked using histograms. Continuous and categorical variables respectively were described using mean (+- standard deviation) and frequency (percentage). Comparison of variables was done using $t$ test for parametric data and Wilcoxon signed rank test for nonparametric data. Statistical significance was defined as a 2-tailed p value of less than 0.05 .

\section{Results}

In this study, we included 113 eyes of 113 subjects who underwent PPV, PPL and SFIOL implantation. The mean age of the study population was $51.5+15.7$ years (median = 55 years; range, $7-80$ years) and 86 were males and 27 females. We have summarised the demographic profile and clinical presentation of the study subjects in Table 1. None of the patients had adequate capsular support for insertion of a posterior chamber IOL in the capsular bag. Preoperative additional raised IOP was seen in $20.4 \%$ patients and was managed with glaucoma medication.

Table 1

Demographic profile and clinical presentation of study subjects

\begin{tabular}{|llll|}
\hline & & Numbers & Percentage \\
\hline Etiology & Blunt object & 111 & $98.2 \%$ \\
\hline & Sharp object & 2 & $1.8 \%$ \\
\hline Baseline clinical presentation & corneal lamellar tears & 2 & $1.8 \%$ \\
\hline & corneal edema & 9 & $8.0 \%$ \\
\hline & severe anterior segment inflammation & 3 & $2.7 \%$ \\
\hline & partial lens subluxation & 55 & $48.7 \%$ \\
\hline & complete lens dislocation & 58 & $51.3 \%$ \\
\hline & vitritis & 10 & $8.8 \%$ \\
\hline & raised intraocular pressure & 23 & $20.4 \%$ \\
\hline
\end{tabular}

At 6 months, the baseline visual acuity of $1.40+-0.66 \operatorname{logMAR}($ median $=1.48$, Snellen equivalent $=$ $6 / 180$ ) increased to $0.23+0.27$ (median $=0.17$, Snellen equivalent $=6 / 9)(p<0.001)$. Overall, visual acuity improved in $96 / 113$ eyes ( $85 \%)$, did not change in $9 / 113$ eyes ( $8 \%$ ) and worsened in the rest $8 / 113$ 
eyes (7\%). Among the eyes in which VA worsened, 4/8 eyes (50\%) had VA better than or equal to $6 / 60$. Among the rest 4 eyes with $V A<6 / 60,2$ had foveal atrophy, 1 had cystoid macular edema and 1 had bullous keratopathy. Of the eyes which showed unchanged VA $(n=9), 6$ eyes $(66.7 \%)$ had VA better than or equal to $6 / 60$.

Final visual outcomes have been summarised in Table 2. Eyes having VA $<6 / 36$ reduced from $79.6 \%(n=$ $90)$ at baseline to $4.4 \%(n=5)$ at final follow-up $(p<0.001)$. Eyes having VA $>=6 / 12$ increased from $8.8-$ $85.8 \%(p<0.001)$. Patients having VA ranging between $6 / 18$ and $6 / 36$ changed from $11.5-9.7 \%(p<$ $0.001)$. Postoperatively, IOP was found raised in $2 \%$ eyes only and this change was statistically significant $(p<0.001)$. Final spherical equivalent of the eyes was $-0.76+-0.33$ DS.

Table 2

Functional status of study subjects before and after surgery

\begin{tabular}{|llllll|}
\hline \multirow{2}{*}{ Visual acuity (Snellen) } & \multicolumn{2}{l}{ Preoperative } & \multicolumn{2}{l}{ Postoperative } & \multirow{2}{*}{ P value } \\
\cline { 2 - 5 } & No. of eyes & Proportion & No. of eyes & Proportion & \\
\hline $6 / 6$ & 3.00 & $2.7 \%$ & 29 & $25.7 \%$ & $<0.001$ \\
\hline $6 / 9$ & 3.00 & $2.7 \%$ & 48 & $42.5 \%$ & $<0.001$ \\
\hline $6 / 12$ & 4.00 & $3.5 \%$ & 20 & $17.7 \%$ & $<0.001$ \\
\hline $6 / 18$ & 5.00 & $4.4 \%$ & 6 & $5.3 \%$ & 0.75 \\
\hline $6 / 24$ & 4.00 & $3.5 \%$ & 3 & $2.7 \%$ & 0.73 \\
\hline $6 / 36$ & 4.00 & $3.5 \%$ & 2 & $1.8 \%$ & 0.43 \\
\hline$<6 / 36$ & 90.00 & $79.6 \%$ & 5 & $4.4 \%$ & $<0.001$ \\
\hline$>=6 / 12$ & 10.00 & $8.8 \%$ & 97 & $85.8 \%$ & $<0.001$ \\
\hline$>=6 / 36-6 / 18$ & 13 & $11.5 \%$ & 11 & $9.7 \%$ & 0.66 \\
\hline
\end{tabular}

\section{Complications}

The complications noted in all the operated eyes till the last follow-up have been summarised in Table 3 . The most common cause of subnormal vision gain at final follow-up after surgery was commotio retinae and associated macular degeneration because of closed globe injury. Three eyes suffered from retinal detachment at postoperative 1,1, and 3 months respectively after SFIOL surgery, and underwent silicone oil injection. At the final follow-up all the eyes had silicone oil removal done when the final VA was documented. Eyes showing cystoid macular edema were treated with prolonged duration of non-steroidal anti-inflammatory drops with slow tapering. No patient developed intractable glaucoma which was not amenable to topical medication and none required filtration surgery at 6 months. One patient developed 
choroidal neovascularization secondary to choroidal rupture, and required two intravitreal bevacizumab injections.

Table 3

Postoperative complications in operated eyes

\begin{tabular}{|lll|}
\hline Complications noted postoperatively & & $\boldsymbol{p}$ value $=<\mathbf{0 . 0 0 1}$ \\
\hline raised intraocular pressure & 2 & $1.8 \%$ \\
\hline retinal detachment & 3 & $2.7 \%$ \\
\hline cystoid macular edema & 2 & $1.8 \%$ \\
\hline macular degeneration & 8 & $7.1 \%$ \\
\hline subretinal hemorrhage & 1 & $0.9 \%$ \\
\hline choroidal neovascularisation & 1 & $0.9 \%$ \\
\hline corneal opacity & 1 & $0.9 \%$ \\
\hline bullous keratopathy & 2 & $1.8 \%$ \\
\hline
\end{tabular}

\section{Discussion}

Subluxation of crystalline lenses may occur due to trauma or post-surgery and can lead to serious sightthreatening complications like intraocular inflammation, elevated IOP, cystoid macular edema and retinal detachment. Pars plana vitrectomy along with lensectomy has been described as an effective means of removing the lens matter, without the risk of creating unnecessary vitreous traction and iatrogenic break formation. Hence, older intracapsular methods of lens removal may be associated with a higher risk of retinal detachment [4].

Following lensectomy, visual rehabilitation of aphakia becomes important, and among the various options available for such cases are aphakic spectacles, contact lenses and implantation of intraocular lenses (IOL). Aphakia management with anterior chamber lenses and iris-fixated lenses have been described, although various complications like corneal endothelial cell loss, peripheral anterior synechiae (PAS), glaucoma and iris pigment dispersion have been reported. Moreover, iris supported lenses may have limited use considering their inflated prices. Scleral-fixated lenses on the other hand are rid of these angle and iris related controversies, however, effective vitrectomy and proper scleral fixation are essential. Combined vitrectomy with scleral fixation of IOL may lead to rapid visual rehabilitation postoperatively. Vitrectomy also removes all evidence of vitreous in the retropupillary plane, reducing chances of vitreous traction. During the surgery, the anterior chamber can be kept formed and stable by infusion pressure, ensuring optimal visualisation to the surgeon. Moreover, if the eye maintains normal IOP during surgery, the chances of scleral needle passing through the ciliary sulcus accurately is higher [5]. Bading et al also proposed that due to the relative safety of the SFIOL procedure, it should be considered an effective 
procedure in younger patients as well.6 Following in-the-bag PCIOL placement, scleral fixation has been said to be the most physiological position for placement of $\mathrm{PCIOL}$, as this positions the refractive status of the eye close to the nodal point of the eye [1].

In this study, we achieved favourable visual outcomes, with $85 \%$ eyes showing net improvement of visual acuity, and a significant improvement in the overall visual acuity of all eyes $(p<0.001)$, in spite of some complications. There was a significant increase in eyes showing VA better than or equal to $6 / 12$ at final follow-up. Among eyes showing subnormal visual acuity, macular degeneration secondary to commotio retina was the most important cause.

Sutured SFIOL methods have been in vogue for a number of years and internal and external fixation techniques have been described. Two-point fixation of the suture may increase risk of axial tilt of IOL, while 3/4-point fixation methods increases the risk of complications caused by increased intraocular manipulations. Previous studies have shown that suture assisted SFIOL places the IOL forwards or backwards from the sulcus. Moreover, sutured IOLs may suffer from numerous complications, namely, suture breakage, decentration, conjunctival erosion, retinal detachment, suprachoroidal hemorrhage, and endophthalmitis [7]. Knot erosion through conjunctiva may provide a direct route for entry of bacteria. 8 Scleral flaps were described to cover knots and prevent this occurrence.

Sutureless techniques attempt to bury the IOL haptics in scleral tunnels or under scleral flaps. Scharioth GB et al described the first sutureless SFIOL surgery using 24-gauge cannula to fashion two diametrically opposite scleral tunnels where the IOL haptics would be inserted $[9,10]$. The technique followed by us is a modified version of Scharioth et al, and we could attain very good globe stability during surgery with this technique, since majority of manipulations were done inside the vitreous cavity. Small diameter tunnel can prevent wound leakage, provide adequate IOL stability and reduce the wound healing time. Moreover, anterior segment ultrasound and anterior segment optical coherence tomography studies have also showed that even without the usage of glue, scleral tunnels can achieve a leak-free closure $[10,11]$. The scleral groove being away from the choroid, also reduces the risk of choroidal hemorrhage or uveitisglaucoma-hyphema syndrome [12]. Also, none of our eyes showed conjunctival erosion or IOL tilt/ decentration. The incidence of IOL tilt or decentration after suture fixation of IOL has been reported to be $0-17 \%$, while the same following intrascleral fixation is around $3.5 \%[7,8,13-15]$. Another important aspect after combined PPV and SFIOL surgery that all surgeons must be aware of is the occurrence of endophthalmitis, which may be relatively rare and can be managed with timely diagnosis and treatment [16].

The limitation of our study is that the follow-up period is relatively short at 6 months, and probably after a longer follow-up, the astigmatism of the patients may stabilize further, leading to further improvement of visual acuities. Our study included eyes with closed globe injury trauma only, and if we would have included open globe injury traumas as well, the outcomes might have been different and maybe skewed. Probably, the same may be evaluated in a different future study. However, the biggest advantage of this technique is that this is quite simple compared to flap and sutured techniques, and maintains good globe 
integrity with insignificant lens haptic movement in the tunnel. These said advantages need to be actively compared in a later study using different techniques, however, this is again a limitation, since the other techniques are not used at our centre in large volumes. However, the simplicity of our technique has been demonstrated in an easy-to-use simulation model, which may help young learners get adept in this technique [17].

In summary, our technique of SFIOL implantation is associated with good visual outcomes for eyes of closed globe injury presenting with subluxation of lens. Moreover, this technique uses less number of surgical maneuvers, thereby reducing surgical trauma and reducing the learning curve, making it easy to be learned by junior surgeons.

\section{Declarations}

Ethical approval: All procedures performed in this study were in accordance with the ethical standards of the institutional research committee and with the 1964 Helsinki declaration and its later amendments or comparable ethical standards (Aravind Eye Hospital Institutional Research Committee, RET201900207). Informed consent was obtained from all individual participants included in the study.

Source of funds: None

Proprietary interest: None

Conflict of interest: None of the authors declares that he has any conflict of interest

Competing Interests: None

Funding Info: No funds were needed for this study

Author contribution: NBK: concept design, final approval; SS: analysis, drafting manuscript, final approval; GK: data collection, final approval; OR: final approval; KR: final approval

Data Availability: Data is available with the corresponding author on request

Animal Research (Ethics): NA

Consent to Participate (Ethics): Informed consent was obtained from all individual participants included in the study at the time of their treatments performed.

Consent to Publish (Ethics): Informed consent for potential research publication was obtained from all individual participants included in the study at the time of their treatments performed.

Plant Reproducibility: NA

Clinical Trials Registration: NA 


\section{References}

1. Khokhar S, Pillay G, Sen S et al. Clinical spectrum and surgical outcomes in spherophakia: a prospective interventional study. Eye (Lond). 2018; 32:527-536

2. Nb K, Kohli P, Pangtey BPS, Ramasamy K. (2018) Evaluation of sutureless, glueless, flapless, intrascleral fixated posterior chamber intraocular lens in children with ectopia lentis. J Ophthalmol. 29:3212740

3. Kannan NB, Sen S, Damodaran S, Debbarma M, Rajan RP, Kumar K, et al. Sutureless scleral fixated intraocular lens implantation for refractive rehabilitation in eyes with spherophakia. J Vitreoretin Dis. 2020. doi.org/10.1177/2474126420936187

4. Naeser K, Baggesen K, Knudsen EB. Retinal detachment following intracapsular cataract extraction. A 10-year follow-up study. Acta Ophthalmol Scand. 1998;76(6):727-30

5. Althaus C, Sundmacher R. Endoscopically controlled optimization of trans-scleral suture fixation of posterior chamber lenses in the ciliary sulcus. Ophthalmologie 1993;90:317e24.

6. Bading G, Hillenkamp J, Sachs HG, Gabel VP, Framme C. Long-term safety and functional outcome of combined pars plana vitrectomy and scleral-fixated sutured posterior chamber lens implantation. Am J Ophthalmol 2007;144:371e7.

7. Schechter RJ. Suture-wick endophthalmitis with sutured posterior chamber intraocular lenses.. J Cataract Refract Surg. 1990;16:755-6. Heilskov T, Joondeph BC, Olsen KR, Blankenship GW. Late endophthalmitis after transscleral fixation of a posterior chamber intraocular lens. Arch Ophthalmol. 1989; 107:1427

8. Kang HM, Chung EJ. Late-onset Citrobacter koseri endophthalmitis with suture exposure after secondary intraocular lens implantation. Korean J Ophthalmol. 2011; 25:285-288

9. Gabor SG, Pavlidis MM. Sutureless intrascleral posterior chamber intraocular lens fixation. J Cataract Refract Surg. 2007; 33:1851-4.

10. Scharioth GB, Prasad S, Georgalas I, Tataru C, Pavlidis M. Intermediate results of sutureless intrascleral posterior chamber intraocular lens fixation. J Cataract Refract Surg. 2010; 36:254-9

11. Bielinski P, Jasielska M, Wyszynska A, Matequsz Winiarczyk, Mackiewicz. Pars plana vitrectomy with transscleral fixation of posterior chamber lens in the treatment of post-traumatic lens dislocation. Int J Ophthalmol.

12. Piette S, Canlas OAQ, Tran HV et al (2002) Ultrasound biomicroscopy in uveitis-glaucoma-hyphema syndrome. Am J Ophthalmol 133:839-841

13. Smiddy WE, Flynn HW (1993) Needle-assisted scleral fixation suture technique for relocating posteriorly dislocated IOLs. Arch Ophthalmol Chic III 1960 111:161-162

14. Chan CK, Agarwal A, Agarwal S, Agarwal A (2001) Management of dislocated intraocular implants. Ophthalmol Clin N Am 14:681-693 
15. Shakin EP, Carty JB (1995) Clinical management of posterior chamber intraocular lens implants dislocated in the vitreous cavity. Ophthalmic Surg Lasers 26:529-534

16. Kannan NB, Sen S, Mishra C, et al. (2021) Ten-year trends in the incidence, clinical profile and outcomes of acute-onset endophthalmitis following combined pars plana vitrectomy and sutureless, glueless and flapless scleral fixation of intraocular lenses. Int Ophthalmol.

17. Damodaran S, Babu N, Sen S, Arthur D, Ramasamy K. Frugal model for scleral fixated intraocular lens simulation. Indian J Ophthalmol. 2021;69(2):463-464 